Print

a

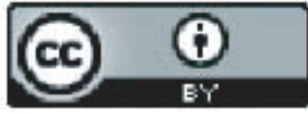

Online

ISSM $2010-6054$

Mediscope

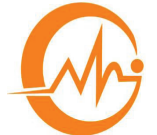

The Journal of GMC

\title{
Modulation of nociception by corticotrigeminal pathway- A narrative review
}

\begin{abstract}
S Ainan ${ }^{1}$
Abstract

Management of chronic pain is one of the most important reason to which medications are given. Traditional medicines which have been used to relieve pain are having a number of limitations. Therefore, novel therapies for pain treatment are essential. Our nervous system can process any kind of injurious stimuli, which is known as nociception. The mechanism of nociception involves a complex interaction of peripheral and central nervous system structures. Brain or cerebral cortex has its own controlling mechanism for pain perception. Trigeminal nerve is the fifth cranial nerve and it receives pain sensation from oro- and craniofacial region and sends the information up to cortex. Recent investigations demonstrate another important role of cortical neurons in addition to pain perception, that is, corticotrigeminal (cortex to trigeminal) pathway excites neurons in the trigeminal nerve that leads to decrease in the pain response induced by noxious stimuli. Thus, as this mechanism can be induced at early stage of nociception, it may reduce the pain sensation. So, the corticotrigeminal pathway could be a new potential target for pain therapies. This short review revisits the concepts how stimulation of primary somatosensory cortex can be transmitted via corticotrigeminal tract which aim for the inhibitory neurons in spinal trigeminal nucleus caudalis $(\mathrm{SpV} c)$ and thus potentially generate a feedforward inhibition, explaining the pain modulatory role of the corticotrigeminal pathway.
\end{abstract}

Key words: Corticotrigeminal pathway, feedforward inhibition, trigeminal spinal caudalis,

\section{Introduction}

The trigeminal sensory nerves comprise primary neuronal cell body situated in the trigeminal ganglion (TG), peripheral axons innervate the corresponding regions of the craniofacial structures and the central terminals make synapse with second order neurons in the trigeminal sensory nuclei (TSN), located in the brainstem. ${ }^{1}$ The TSN is subdivided into the principal or main sensory nucleus, subnuclei oralis (Vo), subnuclei interpolaris (Vi) and subnuclei caudalis
(SpVc). ${ }^{2}$ Among these second order neurons, the $\mathrm{SpV}$ c receives nociceptive afferent inputs activated by innocuous thermal stimuli or noxious stimuli. ${ }^{3}$ There are several common acute and chronic painful ailments affecting the craniofacial region, such as, temporomandibular disorders, toothaches, headaches, and trigeminal neuralgia. ${ }^{3}$ The exact mechanism of pain control in the trigeminal system is still under investigation as per the trigeminal system appears to be greatly different from the spinal system. ${ }^{1}$ 
The SpVc (subnucleus caudalis) owns an intrinsic circuitry for nociceptive processing allowing substantial interaction between the afferent inputs from peripheral tissues and from descending projections from several areas of central nervous system (CNS), including the reticular formation, raphe nuclei, parabrachial area, locus coeruleus, hypothalamus, amygdala, and several areas of the cerebral cortex (e.g. sensorimotor; anterior cingulate; prefrontal). ${ }^{2}$

CNS has an intrinsic well characterized nociceptive modulatory mechanism known as descending pain modulatory circuit (the periaqueductal gray-rostroventral medulla system). ${ }^{4,5}$ This mechanism suppress pain (most commonly by releasing endogenous opioids, e.g. encephalin, endorphins) at initial stages of nociceptive processing in the second order neurons which receive nociceptive afferents as this could ultimately minimize the transmission of pain signals to higher brain areas. ${ }^{3}$

Pain can be described in terms of three hierarchal stages such as, sensory-discriminative, motivational-affective and cognitiveevaluative elements (e.g. thoughts concerning the cause of pain). ${ }^{6}$ The forebrain structures, comprising the amygdala and neocortex has been known to regulate the cognitive aspect of pain, offers an indirect influences over the descending pain modulatory system. ${ }^{7}$ In addition to this indirect influence, it has been observed in some animal models that, dense anatomical projection fibers from the somatosensory cortex directly target second order neurons in the trigeminal nuclei and can alter the sensory processing. ${ }^{8,9}$

One of the first reports of direct projections from cortical areas towards trigeminal sensory nuclei (TSN), in cat was suggested by Brodal et al. ${ }^{10}$ Successive research in cats showed direct inputs from primary somatosensory cortex $(\mathrm{SI})^{11}$ and the secondary somatosensory cortex $(\mathrm{SII})^{12}$ to the subnucleus caudalis $(\mathrm{SpVc})$, that receive primary nociceptive afferents from the head and neck. ${ }^{8}$ In addition, direct inputs to SpVc were also observed in rats and mouse originating from SI, SII, and from the insula. $9,13,14$ Till to date, several studies has reported on how the corticotrigeminal pathway can affect the nociceptive processing in the SpVc. Wang et al. ${ }^{9}$ reported that after suppressing the
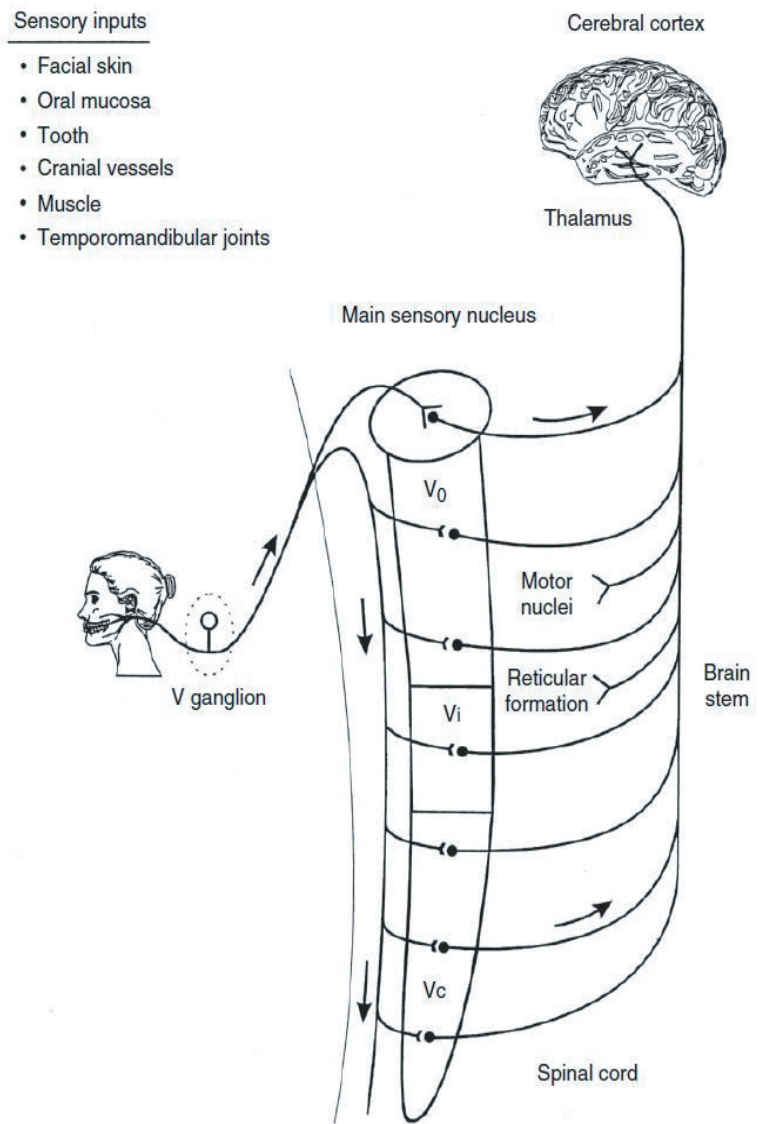

Figure 01: Schema of major somatosensory pathway from the craniofacial region. The cell bodies of primary afferents in the trigeminal nerve are in the trigeminal (V) ganglion and project to second-order neurons in the trigeminal brainstem sensory nuclear complex, which is made up of the trigeminal main sensory nucleus and the trigeminal spinal tract nucleus; consists three subnuclei: Oralis (Vo), interpolaris (Vi), and caudalis(Vc). These neurons may project to neurons at higher levels of the brain. (Adapted from Chichorro et al. ${ }^{3}$ ) 
activity of the corticotrigeminal neurons in insula could decrease the expression of cFOs (a DNA binding protein, commonly used as an activity dependent cell marker along the nociceptive pathway) in SpVc, in a model of neuropathic pain. ${ }^{9}$ Likewise, it has been observed that, stimulation of either primary or secondary somatosensory cortex (SI and SII respectively) can inhibit capsaicin induced firing in SpVc. ${ }^{15}$ Recently, Castro et al.16 have tried their best to address the role of corticotrigeminal circuitry in the modulation of nociception at the level of SpVc and proposed it to be a potential therapeutic target for pain relief. ${ }^{16}$ This group of researchers has shown that, after cortical stimulation, efferents from SI project directly to the target interneurons in

A

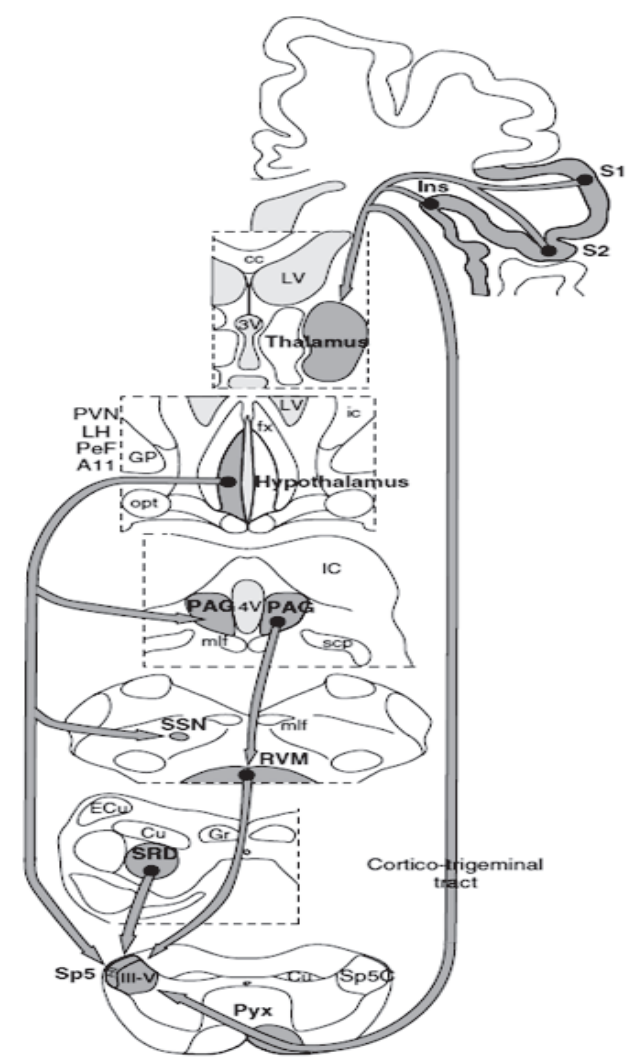

the $\mathrm{SpVc}$ to suppress responses to noxious stimuli and produce behavioral hypoalgesia by feedforward inhibition of projection neurons (exciting the local inhibitory neurons which then inhibit the excitatory projection neurons) through GABAA receptor-mediated signaling. 17

In the aim of developing effective therapeutic approaches against craniofacial pain disorders precise understanding of the trigeminal mechanisms of pain and nociception is indispensible. This review summarizes recent advances in the study of nociceptive processing role of corticotrigeminal pathway in the $\mathrm{SpVc}$, which might contribute to improvements in the existing therapeutic strategies, as well as to the development of novel analgesic therapeutics.

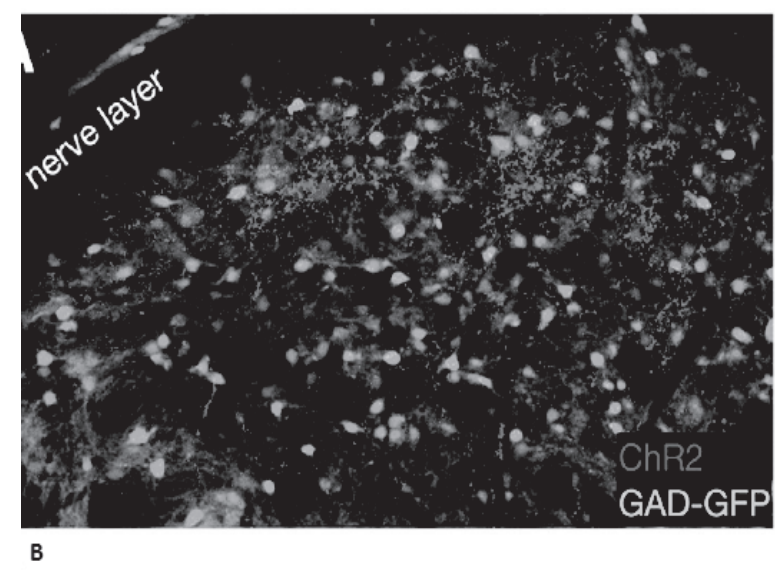

Figure 02: A. The descending pain modulatory system. The widespread source of top-down modulation arises from the cortex to the spinal trigeminal sensory nucleus (Sp5), nucleus caudalis (Sp5C); S1 - primary somatosensory cortex; S2 - secondary somatosensory cortex. (Adapted from Villanueva \& Noseda18). B. mCherry expressing corticotrigeminal axon terminals, following injection of the ChR2-mCherry construct in SI, and GABAergic neurons expressing GAD-GFP in SpVc. "Nerve layer" comprises central and peripheral trigeminal nerve axons. (Adapted from Castro et al.16) 


\section{Materials and methods}

Primary scientific literatures on modulation of nociceptive perception in the TSN b the corticotrigeminal projections was accessed as per journal articles keen to clinical studies and experimental models on craniofacial pain. Online databases were searched using a set of definite search terms and keywords including the ones mentioned in the current text. Databases searched included PubMed $®$, Google scholar ${ }^{\circledR}$, and MEDLINE $®$ resources. Special attention was given to screen and to analyse information on the current concepts incorporating the nociceptive neuronal responses in SpVc and mechanisms of inhibition of noxious stimuli by cortical stimulation in the SpVc to produce analgesia, as well as future research areas delineated in this field.

\section{Discussion}

The spinal trigeminal nucleus caudalis $(\mathrm{SpVc})$ receives projection fibers from the neocortex16 but the nociceptive processing role of this corticotrigeminal pathway in the $\mathrm{SpV}$ c has been under thoroughgoing investigations. So far, various well-designed experiments using anatomical, optogenetic or electrophysiological techniques have been applied in several studies to demonstrate that, descending projections from the primary somatosensory cortex (SI) may modulate the nociception as such stimulation of $\mathrm{SI}$ can attenuate SpVc responses induced by noxious stimuli and reverse the pain hypersensitivity.

Expressions of dense terminal plexus around GABAergic neurons formed by the corticotrigeminal axons in SpVc and In vitro stimulation of these axons excite the interneurons, and create feedforward inhibition of the projections neurons in SpVc.

In several animal models, anatomical tract tracing discovered that corticotrigeminal axonal pathways terminate densely in SpVc, such as, in rats $9,13,14,19$ as well as in cats. ${ }^{11,12,20}$ In one recent report, Castro and his colleugues. ${ }^{16}$ used transgenic GAD-GFP mice and begun their experiments with the anticipation of existence of anatomical substrate for cortical inputs (SI) in the SpVc. ${ }^{16}$ They injected Cholera toxin subunit B (CTB) into the barrel cortex and showed densely distributed CTB labeled corticotrigeminal axons and varicosities, bordering the soma and dendrites of GFP expressing GABAergic neurons particularly in superficial (I-II) and deep (V-VI) laminae. ${ }^{16}$ This observation suggests that corticotrigeminal axons send dense and powerful inputs to SpVc, including to the inhibitory interneurons of this nucleus.

Furthermore, corticotrigeminal inputs also proposed to send anatomical substrate for feedforward inhibition of SpVc projection neurons. To determine the presence of synapse between SpVc neurons and corticotrigeminal axon terminals, a group of researchers performed in vitro recordings from groups of neurons in the superficial layers(lamina I-II), such as, GFP expressing inhibitory GABAergic neurons; projection neurons (identified by retrogradely transported fluorescent latex beads following injections in parabrachial nucleus) and unidentified neurons (labeled with neither beads nor GFP). ${ }^{17}$ As expected, the optogenetic stimulation of corticotrigeminal axons produced strong excitation in inhibitory neurons and as because there was small variation in latency, indicating evocation of monosynaptic responses. ${ }^{17}$ It is noteworthy that, these light evoked responses were ended by the administration of glutamate receptor antagonist $C N Q X$ and $A P V$, which again suggest that these responses are arbitrated by glutamate released from corticotrigeminal pathway. ${ }^{16}$ On the other hand, there was weak excitation followed by strong inhibition in projection neurons evident by generation of markedly smaller magnitude of responses in these neurons than that of the GFP. GABAergic neurons. ${ }^{16}$

Previously, Jacquin et al. ${ }^{21}$ has also reported similar feedforward inhibition that strongly modify the activity of projection neurons in trigeminal $(\mathrm{V})$ subnucleus interpolaris (SpVi)in rats. ${ }^{21}$ The site for termination of nociceptive 
afferents lies in the superficial layers (lamina I-II) of spinal dorsal horn where cortical terminals can directly modulate the neuronal activity. ${ }^{22}$ Besides, the substantia gelatinosa (lamina II) is presumed to be play an key modulatory role in nociceptive transmission from periphery to central nervous system. 23 Therefore, from above discussion it is necessarily clear that, cortical inputs from SI can directly modulate neurons and thus the nociceptive processing in the superficial layer of SpVc.

\section{Nociceptive neuronal responses in SpVc can be suppressed by cortical stimulation}

Stimulation of SI results in suppression of suprathreshold responses of SpVc evoked by noxious stimulation to the skin. ${ }^{16}$ Following in vivo single unit recordings from neurons in laminae $\mathrm{V}$ or $\mathrm{VI}$ in $\mathrm{SpVc}$ in anesthetized male rats, both before and during electrical stimulation $(50 \mathrm{~Hz})$ of $\mathrm{SI}$, it was observed that, combined skin stimulation (current injection through subcutaneous wires in buccal region) and electrical stimulation of SI (through implanted electrode) have reduced the magnitude of responses in SpVc neurons. ${ }^{16}$

Wang et al. ${ }^{9}$ and Braz et al. ${ }^{24}$ suggested that nociceptive processing of projection neurons in the deep layer involve inputs from the interneurons of superficial layer of SpVc. ${ }^{9,24}$ Hence, it can be proposed that cortical inputs reduce nociceptive responses of superficial neurons, causing reduced activation of projection neurons that reside in both superficial and deep SpVc.

Earlier, Malmierca et al. ${ }^{14}$ also observed suppressed nociceptive responses in rat SpVc following $\mathrm{SI}$ stimulation $(5 \mathrm{OHz})$ which was found to be blocked by GABAA and glycine receptor antagonists. ${ }^{14}$ These findings are compatible with another finding by Noseda et al. that, chemically evoked cortical spreading depression involving $\mathrm{SI}$ can suppress responses of SpVc neurons. ${ }^{13}$ Conversely, Gojyo et al. ${ }^{25}$ reported that lower frequency stimulation $(10 \mathrm{~Hz})$ of $\mathrm{SI}$ had no effect on formalin-induced changes in immediate early genes expressed by SpVc neurons. ${ }^{25}$

\section{Inhibition of response to noxious stimuli by cortical stimulation produces analgesia}

To be sure, SI stimulation has been shown to inhibit the activity of dorsal horn neurons in rats $^{26}$, cats ${ }^{27}$, and monkeys ${ }^{28}$, and to ameliorate pain in humans. ${ }^{29,30}$ Interestingly, Castro et al. ${ }^{16}$ for the first time, tested and scored the grooming behaviors in rats following the $\mathrm{SI}$ stimulation. After applying $5 \%$ capsaicin cream over the buccal area and three types of grooming responses were observed, such as, (i) rubbing the face with forepaws; (ii) rubbing the cheek and lower lip against cage floor;(iii) scratching the face with hindpaw. The SI stimulation reduces the duration of grooming response was significantly. ${ }^{16}$ This finding reveals that cortical (SI) stimulation via corticotrigeminal inputs to SpVc inhibit the neuronal response evoked by noxious stimuli and thereby reduce the pain perception as well as produce analgesia.

\section{Conclusion}

Until now motor cortex has been given priorities over somatosensory cortex as a potential suppressor of nociception. ${ }^{31}$ To be sure, there are several chronic pain conditions which have been treated by cortical stimulation through subdural electrodes or by using noninvasive transcranial magnetic stimulation. ${ }^{23}$ However, to expand these approaches, a precise knowledge about the effective site of stimulation is inevitable. There is still a huge knowledge gap in the actual nociceptive processing in the trigeminal sensor nuclei (TSN) as it is fairly different from other body parts in structural and pharmacological features. A thorough understanding of corticotrigeminal pathway as an effective modulator of pain perception can be a promising target for therapeutic interventions to alleviate chronic debilitating craniofacial pain. Nevertheless, further investigations are required to find out the precise neuronal 
References

1. Takemura $M$, Sugiyo $S$, Moritani $M$, Kobayashi M, Yonehara N. Mechanism of orofacial pain control in the central nervous system. Arch Histol Cytol. 2006;69(2):79-100.

2. Dubner R, Iwata $K$ and Wei F. Neurophatic orofacial pain mechanisms: Insights from animal models. In: Sessle BJ (ed.) Orofacial pain: Recent advances in assessment management and understanding of mechanisms. Washington DC: IASP Press, 2014.

3. Chichorro JG, Porreca F, Sessle B. Mechanism of craniofacial pain. Cephalalgia. 2017;37(7):613-626.

4. Fields HL. Pain modulation: expectation, opioid analgesia and virtual pain. Prog Brain Res. 2000;122:245-253.

5. Heinricher MM, Tavares I, Leith JL, Lumb BM. Descending control of nociception: Specificity, recruitment and plasticity. Brain Res Rev. 2009;60:214-225.

6. Melzack R, Casey KL. Sensory, motivational and central control determinants of pain: A new conceptual model. In: Kenshalo, D. ed. The Skin Senses. Springfield IL: Charles C Thomas,1968.

7. Tracey I, Mantyh PW. The cerebral signature for pain perception and its modulation. Neuron. 2007; 55:377-391.

8. Dubner R, Ren K. Brainstem mechanisms of persistent pain following injury. J Orofac Pain.2004;8:299-305.

9. Wang J, Li ZH, Feng B, Zhang T, Zhang H et al. Corticotrigeminal projections from the insular cortex to the trigeminal caudal subnucleus regulate orofacial pain after nerve injury via extracellular signalregulated kinase activation in insular cortex neurons. Front Cell Neurosci. 2015;9:1-15.

10. Brodal A, Szabo T, TorvikA. Corticofugal fibers to sensory trigeminal nuclei and nucleus of solitary tract: an experimental study in the cat. J Comp Neurol. 1956;106:527-555

11. Dunn RC Jr, Tolbert DL. The corticotrigeminal projection in the cat:a study of the organization of cortical projections to the spinal trigeminal nucleus. Brain Res. 1982;240:13-25.

12. Tashiro T, Matsuyama T, Higo S. Distribution of cells of origin of the corticotrigeminal projections to the nucleus caudalis of the spinal trigeminal complex in the cat: a horseradish peroxidase (HRP) study. Exp Neurol. 1983;80:178 -185.

13. Noseda R, Constandil L, Bourgeais L, Chalus M, Villanueva L. Changes of meningeal excitability mediated by corticotrigeminal networks: a link for the endogenous modulation of migraine pain. $\mathrm{J} \mathrm{Neu}-$ rosci. 2010;30:14420-14429.

14. Malmierca E, Chaves-Coira I, RodrigoAngulo M, Nuñez A. Corticofugal projections induce long-lasting effects on somatosensory responses in the trigeminal complex of the rat. Front Syst Neurosci. 2014; 8:100.

15. Malmierca E, Martin YB, Nuñe A. Inhibitory control of nociceptive responses of trigeminal spinal nucleus cells by somatosensory corticofugal projection in rat. Neuroscience 2012; 221:115-124.

16. Castro A, Raver C, Li Y, Uddin O, Rubin D et al. Cortical Regulation of Nociception of the Trigeminal Nucleus Caudalis. J. Neurosci. 2017;37:11431-11440.

17. Bista P, Imlach WL. Pathological Mechanisms and Therapeutic Targets for Trigeminal Neuropathic Pain. Medicines (Basel). 2019;6(3).

18. Villanueva L, Noseda R. Trigeminal Mechanisms of Nociception. In: McMahon, S. Koltzenburg, M. Tracey, I. \& Turk, D. ed. Wall and Melzack's Textbook of Pain. Elsevier, 2012. 
19. Smith JB, Watson GD, Alloway KD, Schwarz C, Chakrabarti S. Corticofugal projection patterns of whisker sensorimotor cortex to the sensory trigeminal nuclei. Front Neural Circuits. 2015; 9:53.

20. McHaffie JG, Kruger L, Clemo HR, Stein BE. Corticothalamic and corticotectal somatosensory projections from the anterior ectosylvian sulcus (SIV cortex) in neonatal cats: an anatomical demonstration with HRP and $3 \mathrm{H}$-leucine. J Comp Neurol. 1988;274:115-126.

21. Jacquin MF, Golden J, Rhoades RW. Structure-function relationships in rat brainstem subnucleus interpolaris: III. Local circuit neurons. J Comp Neurol. 1989;282:24-44.

22. Garcia-Larrea L, Peyron R. Motor cortex stimulation for neuropathic pain: from phenomenology to mechanisms. Neuroimage. 2007;37: S71-S79.

23. Pridmore S, Oberoi G, Marcolin M, George M. Transcranial magnetic stimulation and chronic pain: current status. Australas. 13, 258 -265 2005;13:258-265.

24. Braz J, Solorzano C, Wang X, Basbaum Al. Transmitting pain and itch messages: a contemporary view of the spinal cord circuits that generate gate control. Neuron. 2014;82:522-536.

25. Gojyo F, Sugiyo S, Kuroda R, Kawabata A, Varathan V, Shigenaga Y, Takemura M. Effects of somatosensory cortical stimulation on expression of c-Fos in rat medul lary dorsal horn in response to formalininduced noxious stimulation. J Neurosci Res. 2002;68:479-488.

26. Senapati AK, Huntington PJ, LaGraize SC, Wilson HD, Fuchs PN, Peng YB. Electrical stimulation of the primary somatosensory cortex inhibits spinal dorsal horn neuron activity. Brain Res. 2005;1057:134-140.

27. Namba S, Nishimoto A. Stimulation of internal capsule, thalamic sensory nucleus (VPM) and cerebral cortex inhibited deafferentation hyperactivity provoked after gasserian ganglionectomy in cat. Acta Neurochir Suppl (Wien). 1988; 42:243-247.

28. Yezierski RP, Gerhart KD, Schrock BJ, Willis WD. A further examination of effects of cortical stimulation on primate spinothalamic tract cells. J Neurophysiol. 1983;49:424-441.

29. Canavero S, Bonicalzi V, Dotta M, Vighetti $\mathrm{S}$, Asteggiano G. Low-rate repetitiveTMSallays central pain. Neurol Res. 2003;25:151-152.

30. Lee S, Hwang E, Lee D, Choi JH. Pulsetrain stimulation of primary somatosensory cortex blocks pain perception in tail clip test. Exp Neurobiol. 2017;26:90 -96.

31. Saitoh $Y$, Shibata M, Hirano S, Hirata M, Mashimo T, Yoshimine T. Motor cortex stimulation for central and peripheral deafferentation pain: report of eight cases. J Neurosurg. 2000;92:150 -155. 